\title{
Decoloración fotocatalítica del Rojo de Alizarina S empleando nanocatalizadores de hierro
}

\section{Photocatalytic decoloration of Alizarin S Red employing iron nanocatalysts}

\section{Laura C. Lerici}

Centro de Investigación y Tecnología Química (CITeQ-CONICET-UTN), Córdoba - Argentina. Ilerici@frc.utn.edu.ar

\section{Claudio Varela Lopez}

Centro de Investigación y Tecnología Química (CITeQ-CONICET-UTN), Córdoba - Argentina. claudiovarelalopez@gmail.com

\section{Candelaria Leal Marchena}

Centro de Investigación y Tecnología Química (CITeQ-CONICET-UTN), Córdoba - Argentina. cleal@frc.utn.edu.ar

\section{Ariel Vinuesa}

Centro de Investigación y Tecnología Química (CITeQ-CONICET-UTN), Córdoba - Argentina. arielvinuesa@gmail.com

\section{Eliana Diguilio}

Centro de Investigación y Tecnología Química (CITeQ-CONICET-UTN), Córdoba - Argentina. elianadiguilio@outlook.com.ar

\section{María S. Renzini}

Centro de Investigación y Tecnología Química (CITeQ-CONICET-UTN), Córdoba - Argentina. soledadrenzini@gmail.com

\section{Cristian Campos Figueroa}

Facultad de Ciencias Químicas - Universidad de Concepción, Concepción - Chile.

criscamposf@gmail.com 


\title{
Resumen
}

Los colorantes constituyen una clase importante de compuestos orgánicos que encuentran numerosas aplicaciones en nuestra vida cotidiana. El teñido produce efluentes que contienen entre el 10 y 15\% del colorante que finalmente se depositan en los cuerpos de agua. Se sintetizaron y caracterizaron nanotubos de alúmina impregnados con 2, 6 y $10 \%$ de hierro (Fe-NT), para ser empleados en la decoloración fotocatalítica del Rojo de Alizarina S (ARS). Por DRX se verificaron las señales características de la $\gamma-\mathrm{Al}_{2} \mathrm{O}_{3}$. El área superficial disminuyó con el aumento del contenido hierro oscilando entre 87 y $116 \mathrm{~m}^{2} / \mathrm{g}$. La presencia de hierro fue confirmada por Absorción Atómica. Además, por TEM, se confirmó la estructura tipo nanotubo. Los materiales fueron evaluados catalíticamente en la decoloración del ARS obteniendo porcentajes de decoloración del 50\% para el Fe-NT 2\% y 85-90 \% para los nanotubos con 6 y $10 \%$ de hierro, respectivamente.

Palabras Claves: ROJO DE ALIZARINA S; HIERRO, NANOTUBOS; FOTOCATALISIS

\begin{abstract}
Dyes are an important organic compounds that find numerous applications in our daily lives. The dyeing produces effluents that contain between 10 and $15 \%$ of the dye that are finally deposited in the bodies of water. Alumina nanotubes impregnated with 2, 6 and 10\% of iron (Fe-NT) were synthesized and characterized to be used in the photocatalytic degradation of Alizarine Red S (ARS). By XRD, the characteristic signals of $\gamma-\mathrm{Al}_{2} \mathrm{O}_{3}$ were verified. Surface area varying from 87 to $116 \mathrm{~m}^{2} / \mathrm{g}$. The presence of iron was confirmed by Atomic Adsorption. In addition, the nanotube structure of the support was confirmed by TEM. The materials were evaluated catalytically in the degradation of the ARS obtaining percentages of degradation of $50 \%$ for the Fe-NT $2 \%$ and $85-90 \%$ for the nanotubes with 6 and $10 \%$ of iron, respectively.
\end{abstract}

Keywords: ALIZARIN RED S; IRON; NATOTUBE; FOTOCATALYSIS

\section{Introducción y Objetivos}

Los colorantes constituyen una clase importante de compuestos orgánicos que encuentran numerosas aplicaciones en nuestra vida cotidiana. Las industrias textiles modernas consumen alrededor del $80 \%$ de los tintes sintéticos. El teñido produce efluentes que contienen entre el 10 y 15\% del colorante que finalmente se depositan en los cuerpos de agua (Jabeen, Shah, \& Khan, 2017). Entonces, el problema crítico es reducir los niveles de toxicidad a los límites permisibles antes de liberar el tinte a los cuerpos acuáticos. El Rojo de Alizarina S (ARS) es un colorante antraquinónico ampliamente utilizado en la industria de cuero y fibras textiles (Kaur, Sharma, \& Kansal, 2016). Posee un pKa 4.5 y una solubilidad en agua de $77 \mathrm{~g} / \mathrm{L}$ a $20^{\circ} \mathrm{C}$ (Fayazi, Ghanei-Motlagh, \& Taher, 2015). Su estructura química se muestra en la Figura 1. Entre los diversos métodos de tratamiento aplicables, los sistemas de oxidación fotocatalítica se presentan como una alternativa eficaz para la purificación de 
aguas contaminadas empleando como fuente energética luz UV-Vis. En particular, la fotocatálisis heterogénea, a diferencia de la mayoría de los procesos fotoquímicos, puede emplearse para tratar mezclas complejas de contaminantes con la posibilidad de utilización de la radiación solar como fuente primaria de energía (de Andrade et al., 2015; J. Li et al., 2015; Sivagami, Krishna, \& Swaminathan, 2014). Las especies fotoquímicas empleadas son semiconductores que mediante la incidencia de un haz de luz con energía suficiente, son capaces de generar agentes oxido-reductores con suficiente vida media y reactividad para transformar a los contaminantes (inorgánicos y orgánicos) en productos de menor riesgo medioambiental.

Existen diversos materiales con propiedades adecuadas para actuar como fotocatalizadores, tales como los óxidos de titanio $\left(\mathrm{TiO}_{2}\right)$, hierro $\left(\mathrm{Fe}_{2} \mathrm{O}_{3}\right)$, estaño $\left(\mathrm{SnO}_{2}\right)$, zinc $(\mathrm{ZnO})$, y sulfuros de zinc (ZnS) y de cadmio (CdS), entre otros. La búsqueda continua de mejoras para los sistemas fotocatalíticos heterogéneos ha llevado al uso de semiconductores soportados con el fin de incrementar el área específica y así la actividad por sitio activo, además de evitar el fenómeno de sinterización. Entre los soportes comunes reportados en bibliografía, podemos mencionar la $\mathrm{SiO}_{2}$ (H. L. Wang, Liang, \& Jiang, 2011), $\mathrm{Al}_{2} \mathrm{O}_{3}$ (Z. Li, Sheng, Wang, \& Xu, 2013), fibras de vidrio (Erjavec et al., 2016), zeolitas (Kuwahara et al., 2012; Pan et al., 2014), entre otros. La alúmina $\left(\mathrm{Al}_{2} \mathrm{O}_{3}\right)$ es uno de los óxidos más importantes debido a que presenta buena estabilidad térmica, superficie específica elevada, propiedades ácido-base modulables, bajo costo y facilidad de preparación (C. L. Lu et al., 2009). Estas características hacen que este material sea atractivo para aplicaciones de absorbente, catalizador y soporte de catalizadores heterogéneos. Existen escasos reportes en bibliografía de catalizadores soportados sobre alúmina con aplicaciones en la fotodecoloración de compuestos orgánicos (Tzompantzi et al., 2014). No obstante, experiencias previas realizadas en nuestro grupo de investigación, con nanotubos $\mathrm{Al}_{2} \mathrm{O}_{3}$ impregnados con hierro, han mostrado resultados promisorios en la degradación de los contaminantes orgánicos. En este sentido, el empleo de $\mathrm{Al}_{2} \mathrm{O}_{3}$ nanoestrutuctrado no sólo promoverá la estabilización superficial de agregados nanométricos de óxidos de fotoactivos, sino que también podría incrementar la actividad catalítica por efecto de confinamiento al interior de las cavidades porosas del soporte. En el presente trabajo se estudia la decoloración fotocatalítica de ARS mediante el uso de $\gamma-\mathrm{Al}_{2} \mathrm{O}_{3}$ nanotubular impregnada con 2, 6 y 10 \% de hierro. La nomenclatura se define como Fe-NT x $\%$ donde $\mathrm{x}$ representa el contenido de Fe en porcentaje en peso en el catalizador.

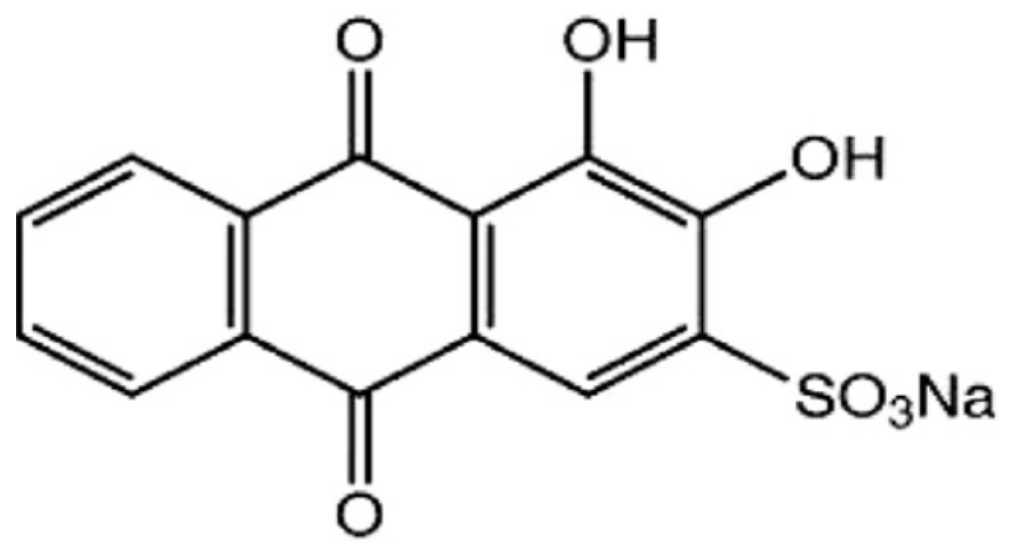

Figura 1. Rojo de Alizarina S. 


\section{Metodología}

La síntesis de la $\mathrm{Al}_{2} \mathrm{O}_{3}$ nanotubular se realizó por método modificado reportado por $\mathrm{Lu}$ y col. (C. L. Lu et al., 2009). Para ello se disolvieron 11,03 g de Al( $\left.\mathrm{NO}_{3}\right)_{3} .9 \mathrm{H} 2 \mathrm{O}$ (Aldrich ${ }^{\circledR}$ ) junto con $1,12 \mathrm{~g}$ de ácido (1R)-(-)camforsulfónico (CSA, Aldrich $\left.{ }^{\circledR}\right)$ como agente surfactante, en 80 $\mathrm{mL}$ de agua destilada y se mantuvo bajo agitación magnética. La mezcla se llevó a pH 5.4 utilizando una solución al 25\% en hidróxido de amonio (Merck $\left.{ }^{\circledR}\right)$, formándose un gel blanco el cual se mantuvo bajo agitación por $10 \mathrm{~min}$. Posteriormente el gel se trasladó a un autoclave de acero inoxidable revestido con teflón y se mantuvo durante $72 \mathrm{~h}$ a $160^{\circ} \mathrm{C}$. Posteriormente se dejó enfriar hasta temperatura ambiente, se procedió a centrifugar el material, luego se lavó en repetidas ocasiones con etanol absoluto y se secó en estufa a $100^{\circ} \mathrm{C}$. Finalmente se calcinó el material a $550^{\circ} \mathrm{C}$ por $4 \mathrm{~h}$, obteniendo un sólido denominado nanotubo (NT).

$\mathrm{La}$ incorporación de hierro se realizó por impregnación húmeda con $\mathrm{FeSO}_{4} .7 \mathrm{H}_{2} \mathrm{O}$ para alcanzar un contenido de Fe (II) de 2, 6 y 10\% en peso. Finalmente, las muestras se secaron a $110{ }^{\circ} \mathrm{C}$ durante la noche y se calcinaron a $500^{\circ} \mathrm{C}$ bajo una atmósfera oxidante durante $8 \mathrm{~h}$.

Los materiales catalíticos fueron caracterizados por la técnica de difracción de rayos $\mathrm{X}$ (DRX) en un equipo Rigaku X-ray Geigerflex, empleando $\mathrm{K}_{\alpha}$ de $\mathrm{Cu}, 40 \mathrm{kV}$ y $2 \mathrm{~mA}$ a un barrido continuo de $0.5^{\circ} \mathrm{min}^{-1}$ y rango $2 \theta$ de $1-90$. Las áreas superficiales ( $\mathrm{S}_{\mathrm{BET}}$ ) de los nanotubos fueron determinadas por BET en un equipo volumétrico Micromeritics Modelo ASAP 2010. El porcentaje de hierro fue determinado por absorción atómica en un espectrofotómetro de absorción atómica 3100 Perkin Elmer. La morfología y el tamaño de los cristales se examinaron mediante microscopía electrónica de transmisión (TEM), en un equipo Philips modelo CM200. Los ensayos de fotodecoloración se llevaron a cabo en un reactor cilíndrico de vidrio provisto de una camisa de refrigeración para mantener la temperatura a $25-30{ }^{\circ} \mathrm{C}$. El reactor fue irradiado por una lámpara de mercurio de alta presión de $125 \mathrm{~W}$ (con un máximo de emisión alrededor de $365 \mathrm{~nm}$ ) que se ubicó en el interior de la camisa refrigerante y se sumergió en la solución. El catalizador se mantuvo en suspensión por medio de agitación magnética y se burbujeó con aire en forma continua durante todo el ensayo. Se retiraron alícuotas de la solución que fueron filtradas y analizadas en un espectrofotómetro Mod T7 DS UV-Vis Marca PERSEE. Según la bibliografía consultada y teniendo en cuenta el $\mathrm{pH}$ de la solución de ARS (6.10), las muestras fueron analizadas a $500 \mathrm{~nm}$ (Kaur et al., 2016) a distintos tiempos de reacción. El tiempo total de reacción fue de 240 minutos y la concentración inicial de ARS y catalizador fue de $50 \mathrm{mg} / \mathrm{L}$ y $500 \mathrm{mg} / \mathrm{L}$, respectivamente. El porcentaje de decoloración de la molécula fue calculado como: Decoloración $(\%)=(1-\mathrm{C} / \mathrm{C} 0)^{*} 100$, donde $\mathrm{C}_{0}$ concentración inicial de ARS y $\mathrm{C}$ la concentración de ARS al tiempo t.

\section{Resultados y Discusión}

Todos los materiales sintetizados han sido caracterizados por DRX tal como se muestra en la Figura 2. En todos los casos se detectan las difracciones típicas para $\gamma-\mathrm{Al}_{2} \mathrm{O}_{3}$ las que no se ven perturbadas por la incorporación de la fase activa. Así mismo se detecta sólo para el catalizador Fe-NT $10 \%$ la difracción a ángulos $2 \theta$ de $32^{\circ}$ atribuida a las fase $\mathrm{a}-\mathrm{Fe}_{2} \mathrm{O}_{3}$ (índice hkl 104) asumiéndose que a contenidos superiores al $6 \%$ en peso de Fe en el soporte se obtienen partículas del óxido superiores a $5.0 \mathrm{~nm}$ que es el límite de detección de la técnica. 


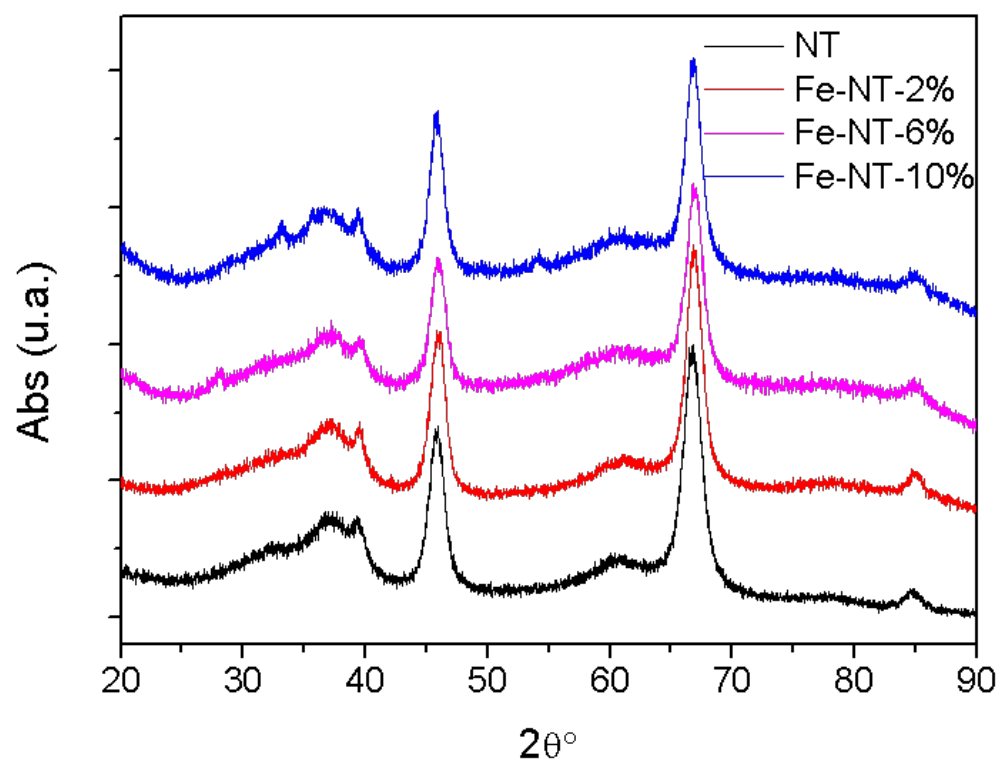

Figura 2. Patrones de difracción del NT y sus expresiones modificadas con Fe.

En la Tabla 1 se presenta, la caracterización fisicoquímica de los catalizadores. Como puede observarse, el aumento del contenido hierro genera una disminución del área superficial de los nanotubos. Este efecto es atribuido al bloqueo que produce la agregación de la fase activa sobre la superficie interna del nanotubo (Gomez et al., 2017). El contenido de Fe en los nanomateriales, determinada por AA, fue muy similar a la cantidad teórica.

\begin{tabular}{|c|c|c|}
\hline Catalizadores & $\mathrm{S}_{\text {BET }}\left(\mathrm{m}^{2} / \mathrm{g}\right)$ & $\mathrm{AA}(\mathrm{Fe} \%)$ \\
\hline $\mathrm{NT}\left(\mathrm{Al}_{2} \mathrm{O}_{3}\right)$ & 188 & - \\
\hline Fe-NT 2\% & 116 & 1.85 \\
\hline Fe-NT 6\% & 105 & 6.10 \\
\hline Fe-NT 10\% & 87 & 9.82 \\
\hline
\end{tabular}

Tabla 1. Caracterización de los catalizadores

A través de las imágenes TEM y HR-TEM, que se muestran en la Figura 3, se observa la estructura tipo nanotubo del soporte con una longitud promedio de $81 \pm 5 \mathrm{~nm}$. La fase activa de los catalizadores también fue caracterizada por TEM y muestra una distribución uniforme y con tamaños inferiores a $5.0 \mathrm{~nm}$ para los sistemas Fe-NT 2\% (en concordancia con los resultados obtenidos por DRX). La imagen de la $\gamma-\mathrm{Al}_{2} \mathrm{O}_{3}$ obtenida por HR-TEM permite confirmar la estructura de nanotubo (Figura 3 d). Si bien no es posible identificar la ubicación de las especies hierro, la gran disminución del área superficial con respecto del soporte sin hierro, nos permite inferir que la fase activa se encuentra ocupando superficie que antes estaba disponible. 


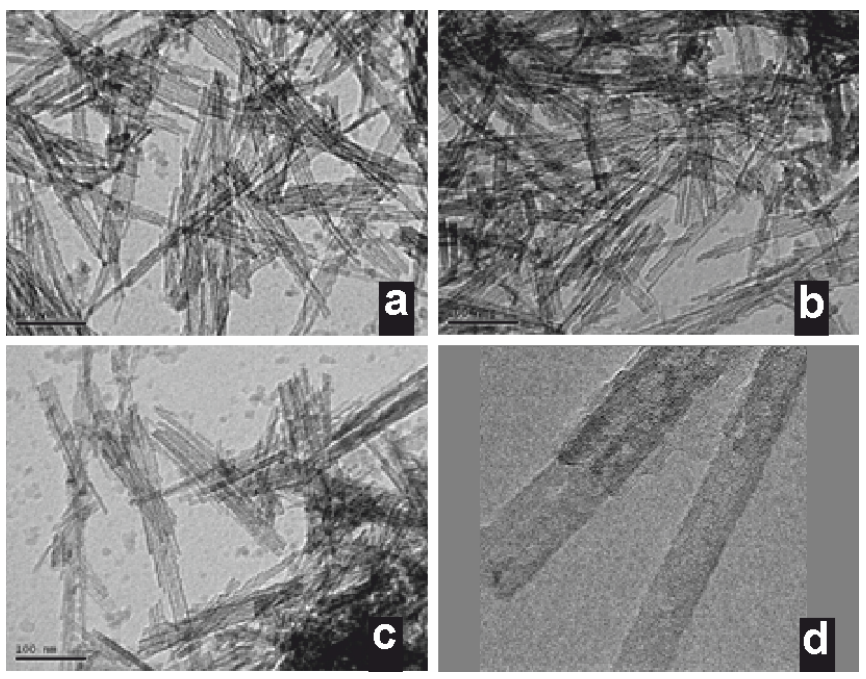

Figura 3. Imágenes TEM: Fe-NT 2\%(a), Fe-NT 6\% (b), Fe-NT 10\% (c) y HR-TEM y-Al2 $\mathrm{O}_{3}$ (d).

El entorno de coordinación de los metales de transición se estudió por DRS UV-Vis. Analizando los espectros correspondientes a las muestras Fe-NT (Figura 4), se observan bandas de absorción significativas que favorece la generación de mayor cantidad de pares hueco-electrón, responsables de promover las reacciones fotocatalíticas (C. Wang \& Huang, 2016). Se pueden diferenciar tres regiones en el rango de 200-300, 300-450 y 450-650 nm. La primera asociada a la transferencia de carga $\left(\mathrm{O} \rightarrow \mathrm{Fe}^{3+}\right)$ entre el hierro y el oxígeno en donde indica que el $\mathrm{Fe}^{3+}$ pudo incorporarse como cationes aislados. Los picos a longitudes de onda $<250 \mathrm{~nm}$ y entre $250-300 \mathrm{~nm}$ aproximadamente, corresponden a la presencia de iones aislados $\mathrm{Fe}^{3+}$ en coordinación tetraédrica y octaédrica, respectivamente (Y. Lu, Zheng, Liu, $\& \mathrm{Mu}, 2007)$. Las energías de band gap de los materiales catalíticos fueron determinadas usando la función de remisión de Kubelka-Munk. Los valores de band gap para los materiales fueron cercanos al reportado en bibliografía para el a-Fe2 $\mathrm{O}_{3}(2,2 \mathrm{eV})$ (Jaafar et al., 2012).

Este resultado es acorde con lo reportado por M. Noorjahan et al. (Noorjahan, Durga Kumari, Subrahmanyam, \& Panda, 2005) que encuentran que con el aumento del contenido de hierro soportado sobre zeolitas $\mathrm{Y}$, los valores de Eg se acercan al del óxido puro. Cabe destacar que los autores reportan un valor máximo de incorporación del metal del 5\% p/p, siendo el contenido de $\mathrm{Fe}$ en las muestras presentadas en este trabajo superior. Este comportamiento se debería al incremento en el número de los niveles de energía que provocan una reducción del band gap. 


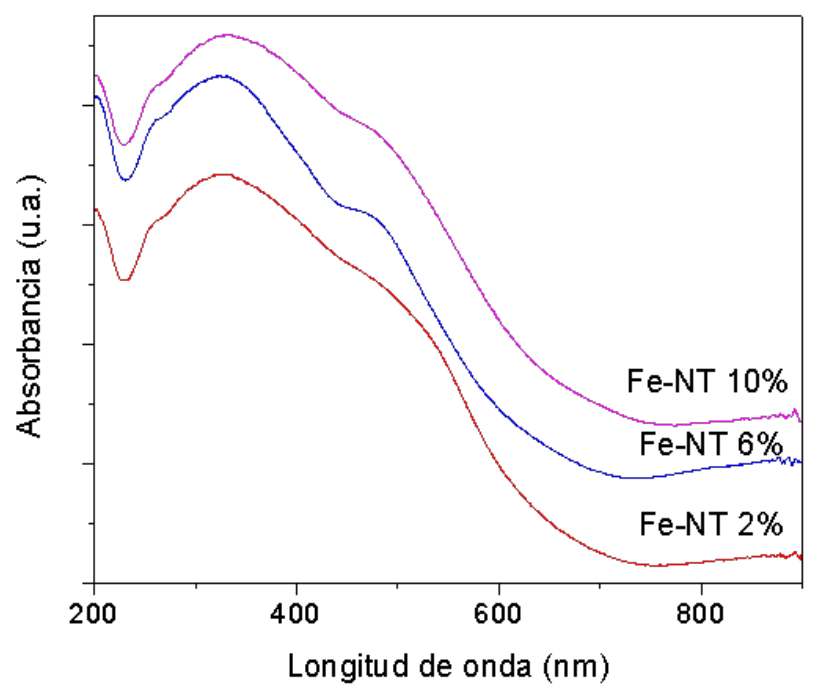

Figura 4. Espectro DRS UV-Vis de los nanomateriales impregnados con hierro.

Se evaluó la decoloración fotocatalítica del ARS sobre Fe-NT con 2, 6 y 10\% de hierro impregnado. La decoloración obtenida fue de 50\% para el ensayo con Fe-NT al $2 \%$, mientras que para los nanomateriales con 6 y $10 \%$ de hierro fue de 85 y $90 \%$, respectivamente (Figura 5). Si bien, el Fe-NT 10\% presento una mejor actividad catalítica, es necesario evaluar la relación costo-beneficio ya que no hay una diferencia marcada en el porcentaje de decoloración.

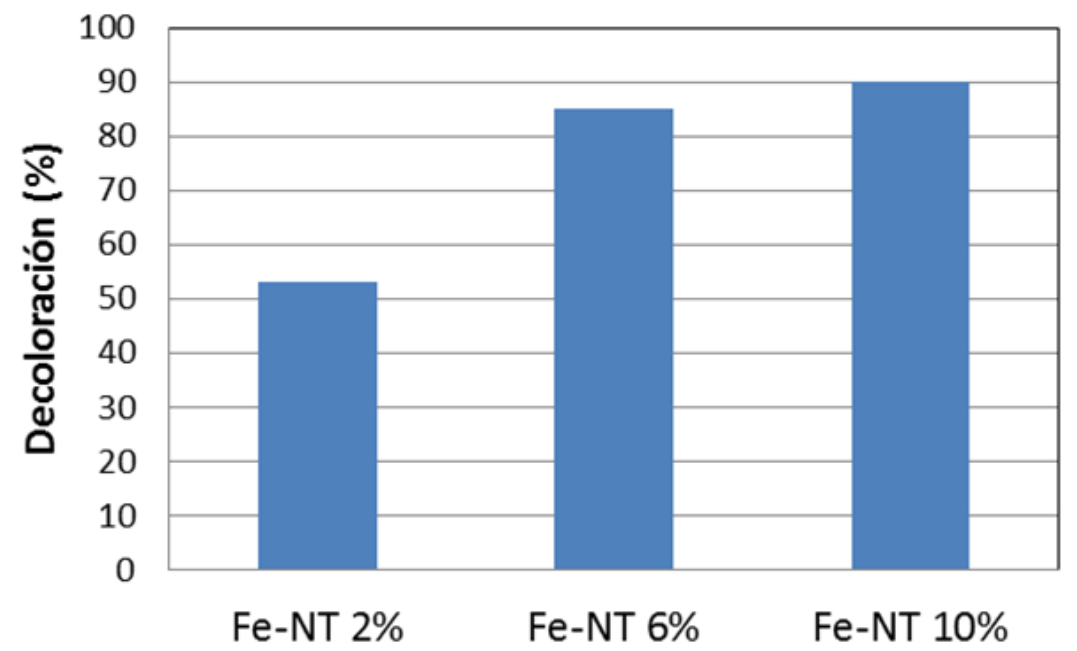

Figura 5. Decoloración fotocatalítica del ARS. 
En la Figura 6, se muestra el espectro de absorción del ARS en la reacción fotodecoloración en presencia de Fe-NT 6\%. Como puede observarse se registró una disminución de la banda a $500 \mathrm{~nm}$ con el tiempo de reacción, con un porcentaje de decoloración mayor al 80 $\%$ los 150 min de reacción. El ensayo en la oscuridad (la solución de ARS + Fe-NT 6\%) mostró una disminución de la concentración del colorante inferior al 20\% efecto atribuido a la adsorción de la molécula sobre la superficie del catalizador. Paralelamente, se realizó una experiencia de fotodecoloración sobre el soporte sin hierro impregnado $\left(\gamma-\mathrm{Al}_{2} \mathrm{O}_{3}\right)$, registrándose una disminución de la concentración del ARS del 19 \%, atribuida a la adsorción de la molécula del colorante sobre el soporte.

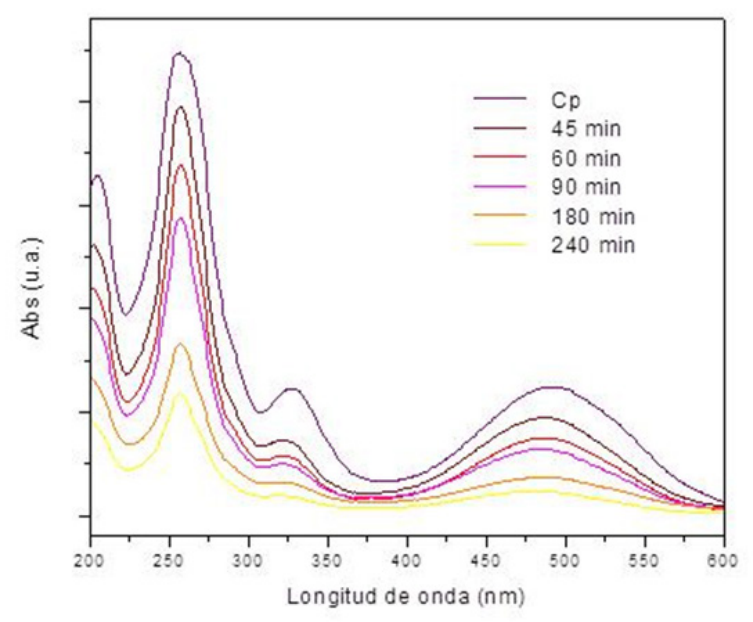

Figura 6. Espectro de absorción de la decoloración fotocatalítica del Rojo de Alizarina debido a la acción del Fe-NT 6\%.

\section{Conclusiones}

Fue posible sintetizar, caracterizar catalizadores de hierro soportados en nanotubos de Al2O3 para la oxidación fotocatalítica de Rojo de Alizarina S empleando una fuente de emisión en el rango del UV. Se lograron porcentajes de decoloración de 85-90 \% para los materiales con 6 y $10 \%$ de hierro, mientras que para el Fe-NT $2 \%$ fue del $50 \%$. El ensayo realizado sobre el soporte sin hierro impregnado demostró la dependencia de la reacción con el contenido de la fase activa. 


\section{Referencias}

de Andrade, F. V., de Lima, G. M., Augusti, R., da Silva, J. C. C., Coelho, M. G., Paniago, R., \& Machado, I. R. (2015). A novel TiO2/autoclaved cellular concrete composite: From a precast building material to a new floating photocatalyst for degradation of organic water contaminants. Journal of Water Process Engineering, 7, 27-35.

Erjavec, B., Hudoklin, P., Perc, K., Tišler, T., Dolenc, M. S., \& Pintar, A. (2016). Glass fiber-supported TiO2 photocatalyst: Efficient mineralization and removal of toxicity/ estrogenicity of bisphenol A and its analogs. Applied Catalysis B: Environmental, 183, 149-158.

Fayazi, M., Ghanei-Motlagh, M., \& Taher, M. A. (2015). The adsorption of basic dye (Alizarin red S) from aqueous solution onto activated carbon $/ \gamma$-Fe2O3 nano-composite: Kinetic and equilibrium studies. Materials Science in Semiconductor Processing, 40, 35-43.

Gomez, S., Lerici, L., Saux, C., Perez, A. L., Brondino, C. D., Pierella, L., \& Pizzio, L. (2017). Fe/ZSM-11 as a novel and efficient photocatalyst to degrade Dichlorvos on water solutions. Applied Catalysis B: Environmental, 202, 580-586.

Jaafar, N. F., Abdul Jalil, A., Triwahyono, S., Muhd Muhid, M. N., Sapawe, N., Satar, M. A. H., \& Asaari, H. (2012). Photodecolorization of methyl orange over a-Fe2O3-supported HY catalysts: The effects of catalyst preparation and dealumination. Chemical Engineering Journal, 191, 112-122.

Jabeen, U., Shah, S. M., \& Khan, S. U. (2017). Photo catalytic degradation of Alizarin red S using $\mathrm{ZnS}$ and cadmium doped $\mathrm{ZnS}$ nanoparticles under unfiltered sunlight. Surfaces and Interfaces, 6, 40-49.

Kaur, S., Sharma, S., \& Kansal, S. K. (2016). Synthesis of ZnS/CQDs nanocomposite and its application as a photocatalyst for the degradation of an anionic dye, ARS. Superlattices and Microstructures, 98, 86-95.

Kuwahara, Y., Aoyama, J., Miyakubo, K., Eguchi, T., Kamegawa, T., Mori, K., \& Yamashita, H. (2012). TiO2 photocatalyst for degradation of organic compounds in water and air supported on highly hydrophobic FAU zeolite: Structural, sorptive, and photocatalytic studies. Journal of Catalysis, 285(1), 223-234.

Li, J., Yang, M., Feng, Z., Lan, F., Teng, C., Li, D., \& Tang, J. (2015). A new HgSe photocatalyst for degradation of organic dyes under visible light irradiation. Materials Letters, 161, 591-594.

Li, Z., Sheng, J., Wang, Y., \& Xu, Y. (2013). Enhanced photocatalytic activity and stability of alumina supported hematite for azo-dye degradation in aerated aqueous suspension. Journal of Hazardous Materials, 254-255(1), 18-25.

Lu, C. L., Lv, J. G., Xu, L., Guo, X. F., Hou, W. H., Hu, Y., \& Huang, H. (2009). Crystalline nanotubes of $\gamma$-AlOOH and $\gamma$-Al2O3: Hydrothermal synthesis, formation mechanism and catalytic performance. Nanotechnology, 20(21).

Lu, Y., Zheng, J., Liu, J., \& Mu, J. (2007). Fe-containing mesoporous silicates with macrolamellar morphology. Microporous and Mesoporous Materials, 106(1-3), 28-34. https://doi. org/10.1016/j.micromeso.2007.02.017 
Noorjahan, M., Durga Kumari, V., Subrahmanyam, M., \& Panda, L. (2005). Immobilized Fe(III)-HY: An efficient and stable photo-Fenton catalyst. Applied Catalysis B: Environmental, 57(4), 291-298.

Pan, Z., Stemmler, E. A., Cho, H. J., Fan, W., LeBlanc, L. A., Patterson, H. H., \& Amirbahman, A. (2014). Photocatalytic degradation of 17a-ethinylestradiol (EE2) in the presence of TiO2-doped zeolite. Journal of Hazardous Materials, 279, 17-25.

Sivagami, K., Krishna, R. R., \& Swaminathan, T. (2014). Photo catalytic degradation of pesticides in immobilized bead photo reactor under solar irradiation. Solar Energy, 103, 488-493.

Tzompantzi, F., Piña, Y., Mantilla, A., Aguilar-Martínez, O., Galindo-Hernández, F., Bokhimi, X., \& Barrera, A. (2014). Hydroxylated sol-gel Al2O3 as photocatalyst for the degradation of phenolic compounds in presence of UV light. Catalysis Today, 220-222, 49-55.

Wang, C., \& Huang, Z. (2016). Controlled synthesis of a-Fe2O3 nanostructures for efficient photocatalysis. Materials Letters, 164, 194-197.

Wang, H. L., Liang, W. Z., \& Jiang, W. F. (2011). Solar photocatalytic degradation of 2-sec-butyl-4,6-dinitrophenol (DNBP) using TiO2/SiO2 aerogel composite photocatalysts. Materials Chemistry and Physics, 130(3), 1372-1379. 